\title{
Modelagem dos Sistemas Térmicos PaRa Motores COM TECNOLOGia FLEX
}

\author{
André Vinicius Oliveira Maggio ${ }^{1}$, Marcos Henrique Carvalho Silva ${ }^{1}$, Armando Antônio M. \\ Laganá $^{1}$ e Joao Francisco Justo Filho ${ }^{1}$ \\ ${ }^{1}$ Escola Politécnica da Universidade de São Paulo \\ E-mails: andremaggio@usp.br, marcoshencarsil@gmail.com, lagana@1si.usp.br, \\ justo@1me.usp.br.
}

\section{RESUMO}

Este artigo propõe modelar a evolução térmica dos diversos blocos do motor. A partir do calor de combustão e da entalpia de entrada dos gases admitidos, procuraremos mapear os diferentes fluxos de energia, dentre eles: energia perdida na fricção do motor, entalpia dos gases de saída, energia efetiva empregada na produção de torque e energia perdida para as paredes do cilindro. De forma a predizer a entalpia dos gases de saída, modelaremos a temperatura de exaustão, estimando seu valor para cada ponto de operação do motor. A energia perdida na fricção do motor e a empregada na produção de torque são obtidas a partir da modelagem do torque. A entalpia de entrada dos gases admitidos e o calor de combustão são obtidas a partir de análise termodinâmica, levando em consideração a temperatura e a relação ar/combustível dos gases. De forma a poder usar o modelo para motores com tecnologia FLEX, iremos analisar como diferentes composições de combustível afetam as formulações apresentadas.

\section{INTRODUÇÃO}

O motor dos veículos a combustão, por mais sofisticados que sejam, são máquinas térmicas que operam convertendo parte da energia proveniente da queima do combustível em trabalho (energia mecânica) e o restante da energia é rejeitada para o ambiente tal como prevê a segunda lei da termodinâmica.

Usando as notações da referência [1], temos a energia da combustão $\dot{m}_{f} \times Q_{L H V}$ alimentando o sistema (figura 1), parte da energia é perdida no gás de escape $\dot{H}_{a}$, nas perdas por atrito de fricção das partes mecânica $\dot{Q}_{\mathfrak{i} f}$ (que engloba também a energia necessária para o funcionamento de dispositivos auxiliares), nas perdas pelo fluido de refrigeração $\dot{Q}_{c}$ e finalmente parte da energia é convertida em energia mecânica $P_{b}$ (energia útil). Parte da energia dos gases de escape $\left(Q_{c, e b}^{*}\right)$ é transferida para o fluido de refrigeração através da carcaça do motor (do inglês: engine block). O restante $\left(\dot{H}_{a}-Q_{e x_{n} \in b}\right)$ é perdido no sistema de exaustão. $Q_{\text {mısc }}$ representa calor cedido ao ambiente advindo da carcaça do motor. O calor $Q_{i f}^{*}$ produzido pelo atrito de fricção é considerado como sendo completamente transferido para a carcaça do motor. Cada parcela será modelada em detalhes nos itens seguintes. 


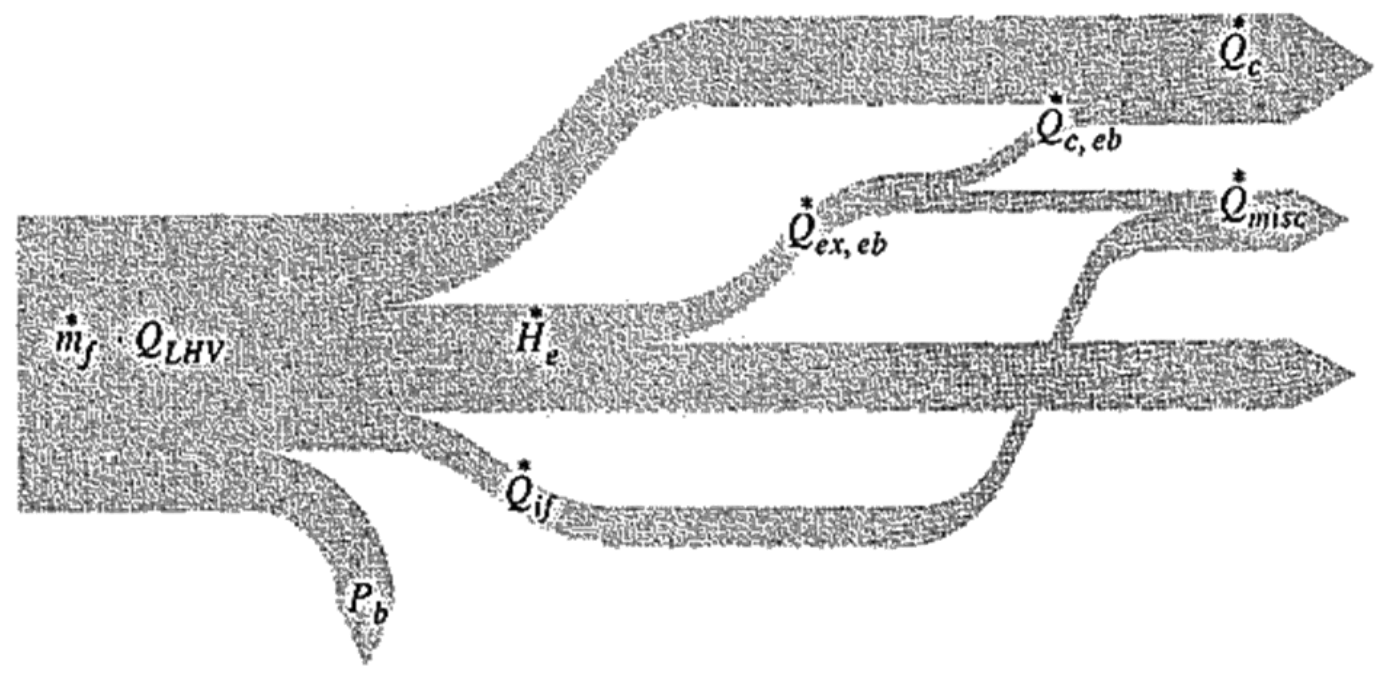

Figura 1: Fluxo de energia em um motor automotivo genérico. Fonte: [1]

\section{ENERGIA NO GÁS DE ESCAPE}

A temperatura dos gases de escape e a sua composição permitem conhecer a entalpia do mesmo. Como a temperatura dos gases de escape é função dos parâmetros da combustão, que podem mudar drasticamente a cada ciclo dependendo das condições impostas como ângulo de ignição, tempo de injeção e taxa de recirculação, a cada ciclo deverá ser corrigido o valor da temperatura dos gases de escape. Em outras palavras, tal temperatura deverá ser uma variável síncrona com período de amostragem de um ciclo, a ser amostrada no centro de exaustão (do inglês: exhaust center). E, devido a esta resposta imediata da temperatura dos gases de escape segundo as condições da combustão, não há interesse em se considerar, na modelagem, um fenômeno transitório nas mudanças desta temperatura [2].

Para medir a temperatura no coletor de exaustão é recomendável um sensor de temperatura como do tipo termopar adequado a operar em altas temperaturas, pois as paredes do coletor de exaustão podem atingir temperaturas da ordem de $900 \mathrm{~K}\left(627^{\circ} \mathrm{C}\right)$ [3], enquanto os gases de escape temperaturas da ordem de $1200 \mathrm{~K}\left(927^{\circ} \mathrm{C}\right)$ [4]. Já a composição dos gases de escape pode ser estimada a partir do conhecimento prévio da mistura de combustível empregada e da relação ar/combustível (fator lambda). Devido à alta eficiência do processo de combustão nos veículos modernos [5], a combustão incompleta será desprezada, considerando apenas que ou o combustível teve combustão completa ou não sofreu combustão, desprezando, portanto, presença de produtos de combustão incompleta ou advindos da reação de mudança de vapor da água. A entalpia dos gases pode, então, ser obtida a partir de tabelas que relacionam a entalpia de cada composto químico com a temperatura.

Com o sensor de temperatura devidamente instalado logo na saída da válvula de escape, sugere-se o levantamento de um mapa base da temperatura dos gases de escape considerando a mistura ar/combustível mais próximo possível da relação estequiométrica (i.e. $\lambda \approx 1$ ), taxa ótima de recirculação e ângulo ótimo de ignição de forma a obter um resultado conforme figura 2. Este mapa poderá sempre ser usado como valor modelado da temperatura dos gases de escape, pois se pretende gastar pouco tempo em excursões de operação distantes da ideal (ângulo ótimo de ignição, relação próxima à estequiométrica e taxa ótima de recirculação) devido a termos perda considerável de eficiência nestas situações. Esta justificativa serve para o caso específico de modelagem da temperatura dos gases de escape, pois estamos apenas 
interessados no efeito cumulativo desta temperatura no motor: Como a constante de tempo de aquecimento do motor é elevada, da ordem de minutos, logo há o interesse apenas em conhecer o quadro genérico da temperatura dos gases de escape e não seu valor preciso a cada ciclo, pois o objetivo final da modelagem de sistemas térmicos é a modelagem da temperatura do motor. $\mathrm{O}$ mapa base da temperatura fica em função da pressão média líquida equivalente (do inglês: brake mean effective pressure) e da velocidade do motor conforme a figura 2.

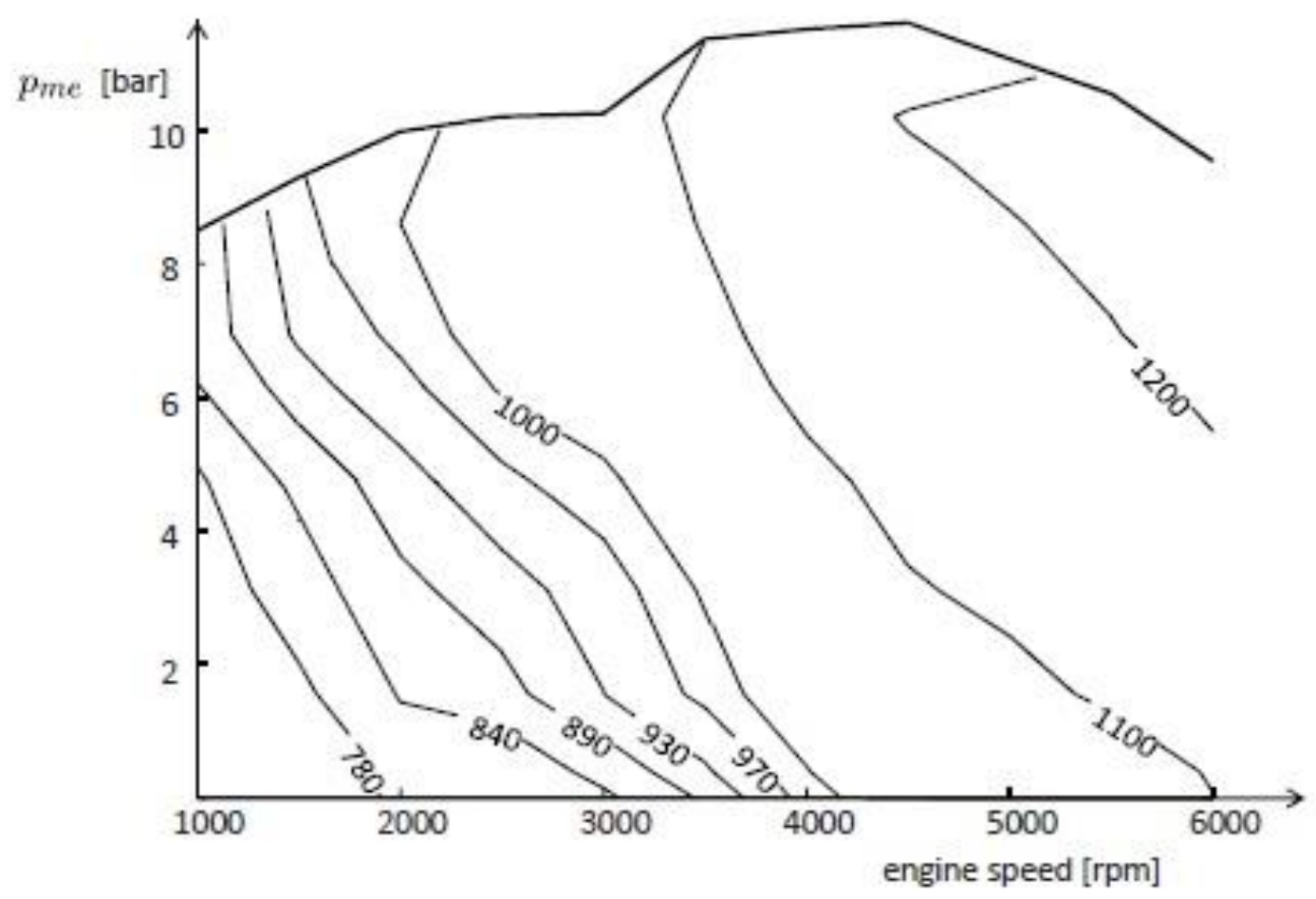

Figura 2: Temperatura dos gases de exaustão $\vartheta_{e g}$ em função da pressão média líquida equivalente e da velocidade do motor. Fonte: [4]

\section{CALOR PERDIDO NO CILINDRO}

Basicamente a energia gerada com a combustão se distribui em cinco parcelas no interior do cilindro: Calor cedido aos gases de exaustão $Q_{\boxminus x h}$, calor perdido no atrito (fricção) das partes mecânicas $Q_{\text {meof }}$, calor perdido devido ao bombeamento (do inglês: pumping) dos gases $Q_{m e o g}$, produção de energia mecânica $Q_{m e}$ e calor perdido nas paredes do cilindro $Q_{g_{s} w}$.

$$
\begin{gathered}
Q_{\text {meof }}=p_{\text {me } 0 f} \times V_{d} \\
Q_{\text {meog }}=p_{\text {me } 0 g} \times V_{d} \\
Q_{m e}=p_{\text {me }} \times V_{d}
\end{gathered}
$$

$p_{\text {me } 0 f}$ é a perda de pressão por fricção.

$p_{m e 0 g}$ é a perda de pressão por bombeamento.

$p_{m e} \quad$ é a pressão media liquida equivalente. 
$V_{d} \quad$ é o volume de deslocamento do cilindro.

$Q_{\text {exh }}$ é a entalpia dos gases de exaustão de um determinado ciclo.

Conhecendo a composição dos gases admitidos pelo cilindro através da taxa de recirculação, composição do combustível utilizado, do ar e dos gases recirculados, e adotando como temperatura aproximada dos gases admitidos a temperatura ambiente, pode-se inferir a entalpia de entrada dos gases admitidos por ciclo $Q_{a d m}$. Aproxima-se, nesta modelagem específica, a temperatura dos gases de admissão pela temperatura ambiente, pois considera-se que há refrigeração dos gases recirculados e que eventual aquecimento do ar admitido devido ao sistema de admissão ou mesmo devido a não refrigeração ideal dos gases recirculados pode ser desprezado por provocar mudanças pouco significativas do valor da entalpia dos gases de admissão.

Usando o princípio da conservação da energia é possível obter o calor perdido para as paredes do cilindro $Q_{g_{l} w}$ :

$$
\begin{aligned}
& \sum_{\text {Ciolo }} \text { Energia entrada }=\sum_{\text {Ciolo }} \text { Energia saída } \\
& Q_{a d m}=Q_{g, w}+Q_{a x h}+Q_{m e}+Q_{m e o g}+Q_{\text {meof }} \\
& Q_{g, w}=Q_{a d m}-Q_{e x h}-Q_{m e}-Q_{m e o g}-Q_{m e o f}
\end{aligned}
$$

\section{MODELAGEM ATRAVÉS DE CÁLCULOS COM O USO DO CICLO TERMODINÂMICO}

Para este tipo de modelagem é aconselhável o uso do sensor de pressão interna ao cilindro, que permite medir a pressão em função do ângulo do virabrequim $p(\Phi)$. Já o volume em função do ângulo do virabrequim $V(\Phi)$ pode ser obtido através do conhecimento prévio da geometria do motor. Por fim aplicando uma mudança de variável nos dois gráficos é possível encontrar a pressão em função do volume $p(V)$, que é o diagrama $\mathrm{PxV}$ do ciclo termodinâmico real.

$\mathrm{Na}$ ausência ou indisponibilidade em se haver um sensor de pressão interna ao cilindro, este pode ser substituído pelo uso do ciclo termodinâmico Otto ideal. Porém, como é possível ver na figura 3, o ciclo ideal pode-se distanciar do ciclo real consideravelmente [6], devendo esta abordagem ser utilizada em último caso.

Uma forma de atenuar esta diferença é corrigir o gráfico segundo um fator $\mathrm{K}_{\mathrm{e}}$, conforme equação (6) e (7). Esta correção baseia-se na asserção que a diferença de pressão entre o ciclo de expansão e o ciclo de compressão para um mesmo valor de volume (uma mesma linha vertical, uma mesma abscissa) é proporcional a eficiência da combustão [4]. Logo, ao aproximarmos a dinâmica da combustão incompleta com a dinâmica de outras perdas, como a dinâmica das transferências do calor, infere-se que (7) é uma aproximação aceitável. Obviamente a curva $\mathrm{p}(\mathrm{V})$ real será diversa da modelada em (7), apresentando morfologia discrepante, devido aos diversos fenômenos de perda terem suas dinâmicas distintas. Porém, 
pode-se usar esta aproximação caso não se disponha da devida instrumentação, em razão de eventuais erros serem equiponderados pelo método de identificação utilizado.
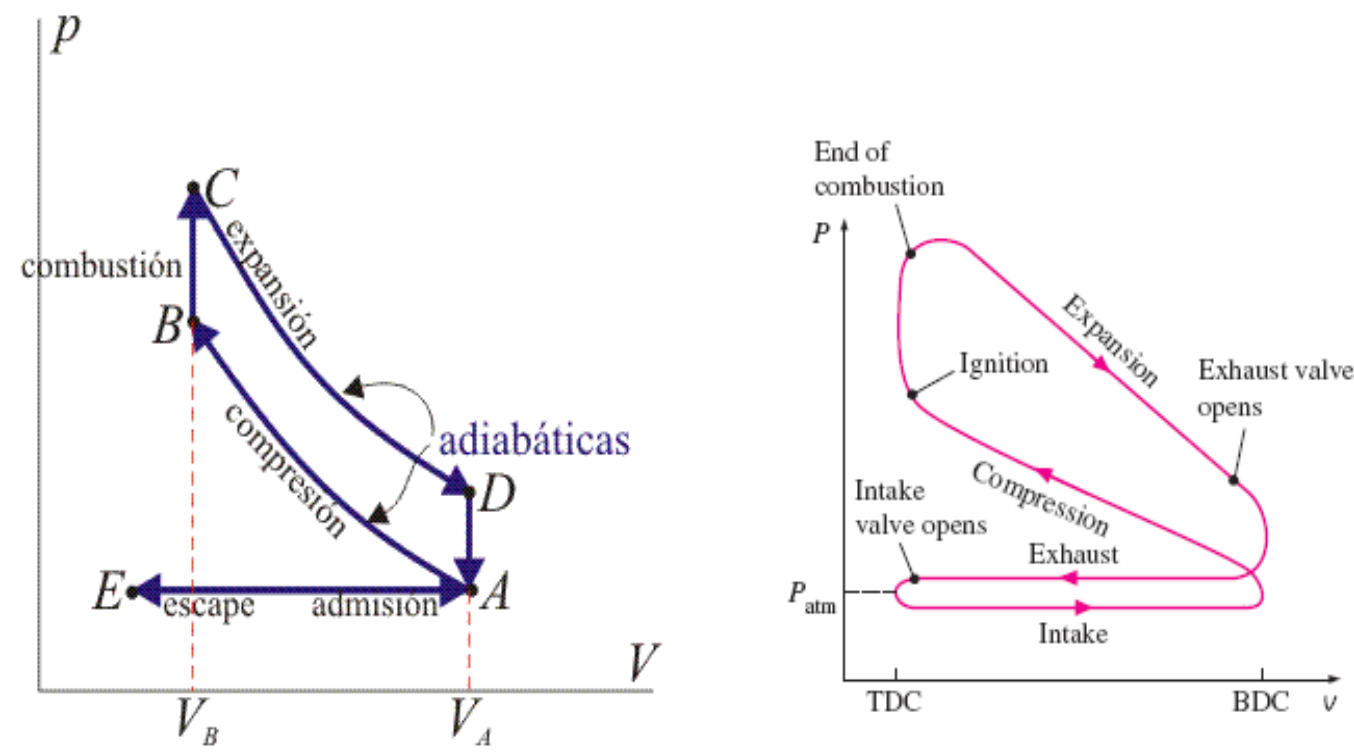

Figura 3: Ciclo otto ideal a esquerda e ciclo real a direita Fonte: [6].

$$
K_{e}=\frac{e(.)}{e_{\text {ottoifienl }}(.)}
$$

$e($.$) é a eficiência modelada, função de diversos parâmetros do motor. A modelagem da$ eficiência pode ser encontrada em referências de modelagem de torque e de sistemas mecânicos de motores a combustão interna, como [4].

$$
p(V)=K_{e} \times p_{\text {otto } 0_{\text {idRaV }}}(V)
$$

\subsection{Modelagem do calor perdido para as paredes do cilindro através do uso do ciclo termodinâmico}

$\mathrm{O}$ calor transferido dos gases para as paredes internas do cilindro $Q_{g, w}$ pode ser calculado por [7]:

$$
Q_{g, w}=\alpha \cdot A \cdot\left(\overline{\vartheta_{g}}-\overline{\vartheta_{w}}\right)
$$

Aonde,

$Q_{g, W}$ é o calor dos gases para a parede do cilindro;

$\alpha \quad$ é o coeficiente de transferência de calor;

$A \quad$ é a área relativa de contato entre os gases e a parede do cilindro;

$\overline{\vartheta_{a}} \quad$ é a temperatura média dos gases;

$\frac{a}{\vartheta_{w}} \quad$ é a temperatura média da parede do cilindro. 
O coeficiente de transferência de calor e a temperatura da parede do cilindro possuem fórmulas empíricas conforme abaixo [7], [8] e [9]:

$$
\alpha=130 \times V_{d}^{-0.06} \times p^{-0.8} \times{\overline{v_{g}}}^{-0.4} \cdot\left(\bar{v}_{p}+1.4\right)^{0.8}
$$

$\bar{v}_{p} \quad$ é a velocidade média do pistão.

$p \quad$ é a pressão instantânea interna ao cilindro.

$$
\overline{\vartheta_{w}}=360+9 \times \lambda^{0.4} \times\left(\omega_{e} \times B\right)^{0.5}
$$

\subsection{Modelagem da temperatura no coletor de escape através do uso do ciclo termodinâmico}

Como o diagrama PxV do ciclo termodinâmico pode ser tido como conhecido, devido a ter sido discutido neste artigo, podemos obter, então, na falta de instrumentação adequada, a temperatura e a pressão dos gases de escape através deste diagrama.

Como a temperatura no centro de exaustão dos gases reservados no cilindro pode ser aproximada como igual à temperatura dos gases reservados no coletor de escape, devido a considerarmos que houve tempo suficiente da abertura da válvula até o centro de exaustão para essas duas temperaturas se aproximarem, devido ao fluxo dos gases de escape formar no centro de exaustão uma linha isotérmica entre o interior do cilindro e o interior do coletor de escape, podemos usar a pressão e o volume do diagrama PxV no centro de exaustão para calcular a temperatura deste centro e usar este valor como temperatura dos gases de escape. Obviamente que, neste raciocínio, perdas foram desprezadas como as ocorridas nas válvulas de escape [7].

\section{MODELO TÉRMICO INTERNO AO MOTOR}

Conforme a Figura 4, parte do calor dos gases de escape é absorvido pela carcaça do motor $\left(Q_{\theta x_{n} \in b}\right)$, elevando sua temperatura. Esse calor pode ser calculado por:

$$
Q_{e x_{i} \theta b}=A_{e x_{i} \theta b} \times \alpha_{\theta x_{i} \theta b} \cdot\left(\vartheta_{e x h}-\vartheta_{\theta b}\right)
$$

$A_{\theta x_{n}, \theta b}$ é a área de contato dos dutos de saída com a carcaça do motor;

$\alpha_{e x, e b}$ é o coeficiente de transferência de calor deste caso específico;

$\vartheta_{e x h}$ é a temperatura dos gases de exaustão nos dutos de saída (pode ser aproximada pela temperatura no coletor de escape);

$\vartheta_{a b} \quad$ é a temperatura média na carcaça do motor.

$\alpha_{\theta x_{i}, b b}$ bem como outros o coeficiente de transferência de calor, para um dado motor genérico, pode ser obtido a partir da relação empírica abaixo [4]:

$$
\alpha= \begin{cases}28.6+4 \times v_{g} & \text { para } v_{g}<5 \mathrm{~m} / \mathrm{s} \\ 21 \times v_{g}^{0.52} & \text { para } v_{g} \geq 5 \mathrm{~m} / \mathrm{s}\end{cases}
$$


$v_{g} \quad$ é a velocidade relativa de fluxo entre as duas superfícies. Para $\alpha_{e x_{i} e b}, v_{g}$ é a velocidade dos gases no duto de saída.

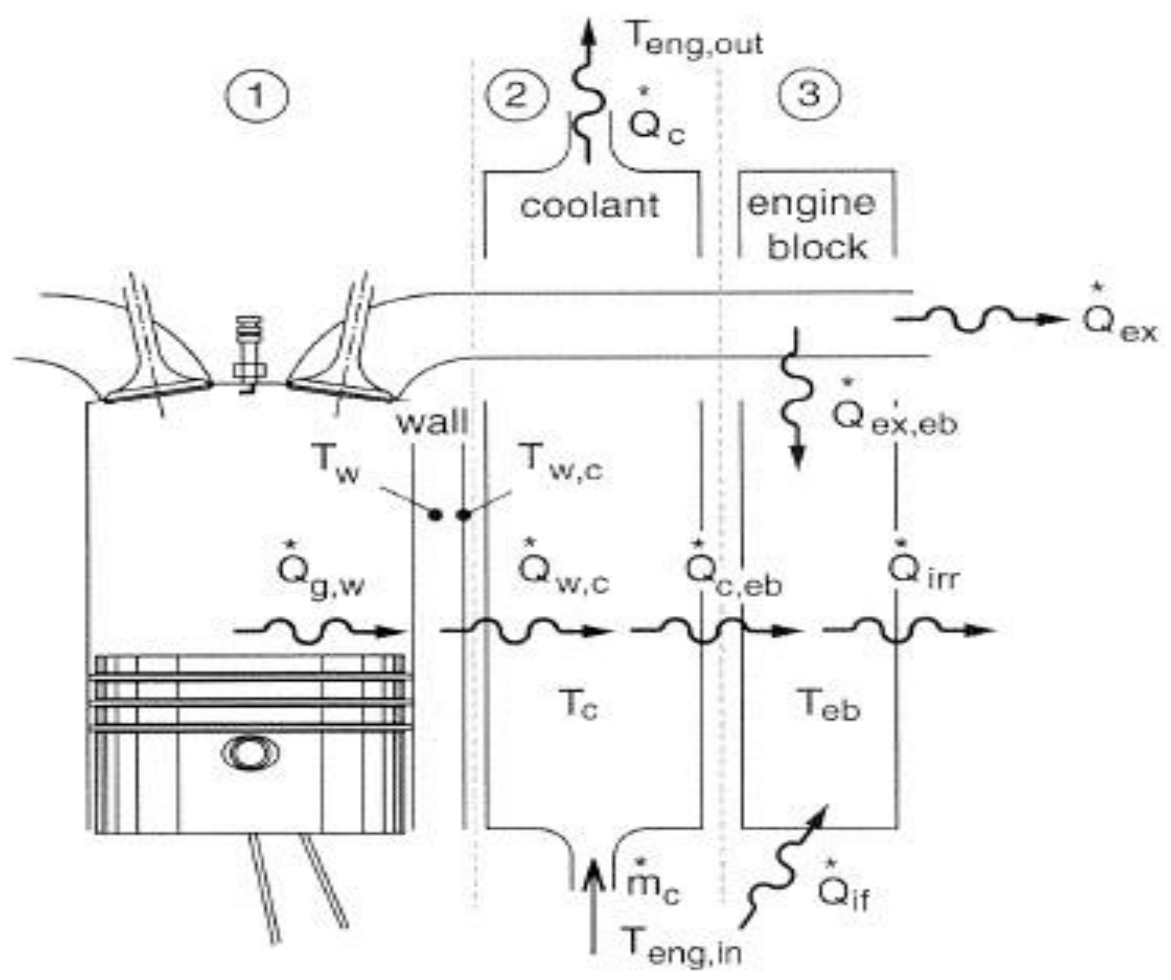

Figura 4: Fluxo de calor dentro do motor. Fonte: [1]

A temperatura na superfície da parede do cilindro $\left(\vartheta_{w, c}\right)$ pode ser aproximada pela temperatura da própria parede do cilindro $\left(\vartheta_{w}\right)$ (Figura 4$)$, considerando que estamos modelando a parede do cilindro como um sistema de parâmetros agrupados. Temos, portanto, $\overline{\vartheta_{w, c}}=\overline{\vartheta_{w}}$, com $\overline{\vartheta_{w}}$ conhecido da equação (10).

$$
\begin{aligned}
Q_{w, c}^{*} & =A_{c} \times \alpha_{c} \times\left(\overline{\vartheta_{w, c}}-\vartheta_{c}\right) \\
\vartheta_{c} & =\frac{\vartheta_{\text {eng,out }}+\vartheta_{\text {eng,in }}}{2}
\end{aligned}
$$

$A_{c} \quad$ é a área da superfície da parede do cilindro em contato com o fluido de refrigeração

$\alpha_{c} \quad$ é o coeficiente de transferência de calor da parede do cilindro para o fluido de refrigeração. Pode-se utilizar a equação (20) para obtê-lo, considerando $v_{g}$ como a velocidade do fluxo do fluido de refrigeração, a ser modelada em seção posterior.

$\vartheta_{c} \quad$ é a temperatura média da porção do fluido de refrigeração interno ao cilindro $\left(\vartheta_{\text {eng,m }}\right.$, usada na equação (3.19));

$\vartheta_{\text {eng out }}$ é a temperatura do fluido de refrigeração na saída do motor.

$\vartheta_{\text {eng,in }}$ é a temperatura do fluido de refrigeração na entrada do motor.

O calor transferido do fluido de refrigeração para a carcaça do motor $\left(Q_{\mathcal{C}_{b} \theta b}^{*}\right)$ pode ser obtido da mesma forma. 


$$
Q_{c_{b} B b}^{*}=A_{\theta b} \times \alpha_{\theta b} \times\left(\vartheta_{c}-\vartheta_{\theta b}\right)
$$

$A_{e b} \quad$ é a área de contato entre o fluido de refrigeração e a carcaça do motor.

$\alpha_{e b}$ é o coeficiente de transferência de calor. Pode-se utilizar a equação (20) para obtê-lo, considerando $v_{g}$ como a velocidade do fluxo do fluido de refrigeração.

O calor cedido da carcaça do motor ao ambiente $\left(Q_{B b, a}^{*}\right)$ ocorre via convecção e irradiação térmica:

$$
Q_{\theta b, a}^{*}=Q_{i r r}^{*}=\sigma \cdot A_{\theta b, a} *\left(\vartheta_{\theta b}^{4}-\vartheta_{a m b}^{4}\right)+A_{\theta b, a} * \alpha_{\theta b, a} *\left(\vartheta_{\theta b}-\vartheta_{a m b}\right)
$$

$\sigma \quad$ é a constante de Stefan-Boltzmann;

$A_{e b, a}$ é a área de contato entre a carcaça do motor e o ambiente;

$\vartheta_{a m b}$ é a temperatura ambiente;

$\alpha_{e b, a}$ é o coeficiente de transferência de calor. Pode-se utilizar a equação (20) para obtê-lo, considerando $v_{g}$ a velocidade do veículo.

Conforme [4] e [1] o fluxo de calor gerado pelo atrito na friç̧ão das partes mecânicas $\left(Q_{i f}^{*}\right)$ pode ser equacionado como:

$$
\dot{Q}_{\mathrm{if}}=p_{\text {meof }} \times V_{d} \times \frac{\omega_{B}}{4 \times \pi}
$$

$p_{\text {meof }}$ é a perda de pressão por fricção.

$\mathrm{O}$ aumento da temperatura dos constituintes do sistema térmico será calculado através das capacidades caloríficas destes elementos. O aumento da temperatura do bloco do motor $\Delta \overline{\vartheta_{w e}}$ devido ao calor por este recebido pode ser calculado através da equação (18). $\mathrm{O}$ fluxo energético absorvido pela parede do cilindro é o fluxo advindo dos gases de combustão internos ao cilindro subtraído do fluxo da parede do cilindro ao líquido refrigerador. Como supõe-se que o material das paredes do cilindro é o mesmo da carcaça do motor, far-se-á $c_{e b}=c_{w}$. Para o fluido de refrigeração, recomenda-se o uso da capacidade calorífica da água $c_{\text {agua }}=c_{c}$. Para o óleo lubrificante, recomenda-se o uso conforme tabela 1, devido à capacidade calorífica do óleo degradado (óleo usado) ser próxima à capacidade calorífica do óleo virgem para todos os tipos de óleo com exceção do óleo mineral sem aditivo [10]. Conforme raciocínio empregado na equação (18), segue a evolução térmica do líquido refrigerador (equação (19)) e a evolução térmica da carcaça do motor (equação (20)). Nesta equação, aproximou-se a constituição do motor como parte advinda da estrutura metálica e parte advinda do óleo lubrificante.

$$
Q_{g, w}^{*}-Q_{w, c}^{*}=m_{w}{ }^{*} c_{w} \times \Delta \overline{\vartheta_{w}}
$$

$m_{w} \quad$ é a massa do bloco do motor.

$c_{w} \quad$ é a capacidade térmica do bloco do motor.

$$
\dot{Q}_{c}=\dot{m}_{c} \times c_{c} *\left(\vartheta_{\text {eng,out }}-\vartheta_{\text {eng,in }}\right)
$$

$\dot{m}_{c} \quad$ é o fluxo mássico do líquido refrigerador. 


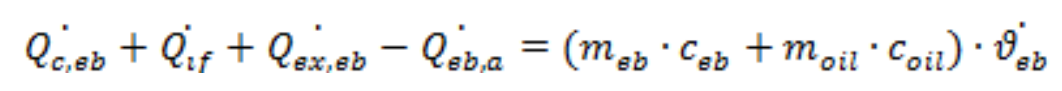

$m_{a b} \quad$ é a massa da estrutura metálica da carcaça do motor.

$m_{\text {oil }}$ é a massa de óleo lubrificante interna à estrutura da carcaça do motor.

Como a massa de líquido refrigerador interna ao motor $m_{c}$ independe do fluxo, podemos calcular a derivada temporal da temperatura média do líquido refrigerador usando uma abordagem diferente da equação (19). Na nova abordagem, procuramos computar a diferença no espectro de temperatura do líquido refrigerador interno ao motor num determinado instante, considerando que o fluxo energético deste instante é a causa deste espectro, conforme equação (21) abaixo:

$$
\dot{Q}_{w, c}^{*}-Q_{c, i b b}^{*}-\dot{Q}_{c}=m_{c}{ }^{*} c_{c} \cdot \dot{\vartheta}_{c}
$$

\begin{tabular}{ccccccccc}
\hline Óleos & \multicolumn{7}{c}{$\mathrm{Cp}\left(\mathrm{J} \mathrm{g}^{-1}{ }^{\circ} \mathrm{C}^{-1}\right)$} \\
lubrificantes & $40^{\circ} \mathrm{C}$ & $60^{\circ} \mathrm{C}$ & $80^{\circ} \mathrm{C}$ & $100^{\circ} \mathrm{C}$ & $120^{\circ} \mathrm{C}$ & $140^{\circ} \mathrm{C}$ & $160^{\circ} \mathrm{C}$ & $180^{\circ} \mathrm{C}$ \\
\hline Mineral & 2,534 & 2,865 & 3,069 & 3,101 & 3,648 & 4,052 & 4,689 & 5,458 \\
Mineral sem aditivo & 2,329 & 2,661 & 2,832 & 2,867 & 3,345 & 3,724 & 4,297 & 4,986 \\
Sintético & 2,382 & 2,714 & 2,890 & 2,944 & 3,430 & 3,825 & 4,424 & 5,122 \\
Semi-sintético & 2,316 & 2,675 & 2,861 & 2,894 & 3,360 & 3,732 & 4,314 & 5,015 \\
\hline
\end{tabular}

Tabela 1: Capacidade calorífica dos óleos lubrificantes não degradados. Fonte: [10]

\section{MODELO TÉRMICO EXTERNO AO MOTOR}

Conforme a Figura 5, parte do calor gerado pelo motor é rejeitado para o ambiente através do radiador.

O calor cedido ao radiador advindo do líquido refrigerador pode ser calculado como:

$$
\dot{Q}_{r i}=c_{c} \times \dot{m}_{r} \times\left(\vartheta_{r i}-\vartheta_{r o}\right)
$$

$c_{c} \quad$ é a capacidade térmica do fluido de refrigeração, o radiador este cheio com este fluido e como a capacidade térmica do radiador é bem menor que a do fluido a capacidade térmica do sistema é aproximadamente igual à do fluido.

$\dot{m}_{r} \quad$ é a fração do fluxo do líquido refrigerador que passa pelo radiador, a ser identificado posteriormente.

$\vartheta_{r i} \quad$ é a temperatura do líquido refrigerador adentrando no radiador.

$\vartheta_{r o} \quad$ é a temperatura do líquido refrigerador saindo do radiador. 


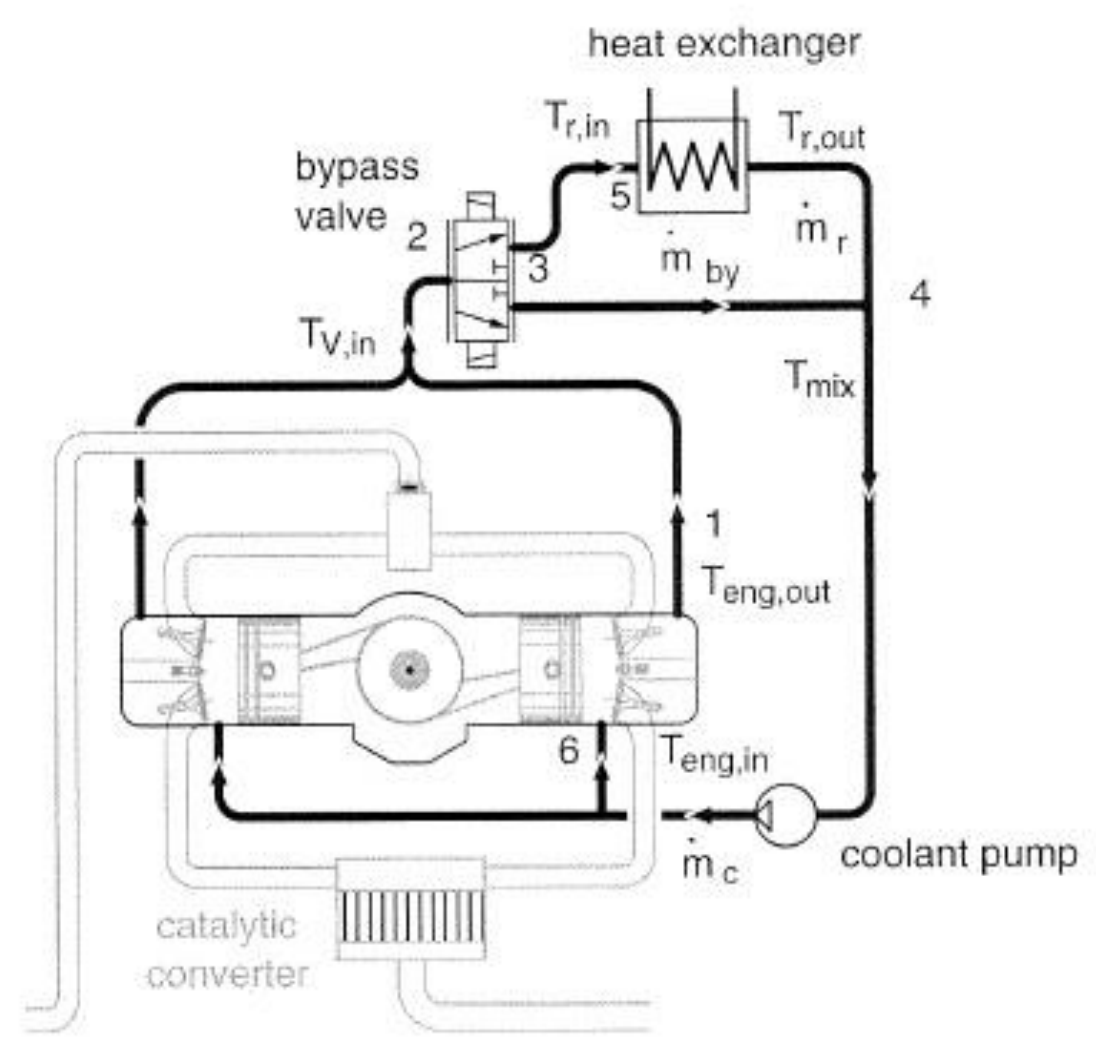

Figura 5: fluxo de calor na região externa do motor. Fonte: [1]

O calor por convecção que se transfere do radiador para o meio ambiente (note que estamos desconsiderando a transferência de calor por irradiação) pode ser calculado como:

$$
Q_{r o}^{*}=\alpha_{r}\left(v_{v}\right) \cdot A_{r} \cdot\left[\vartheta_{r}-\vartheta_{\alpha}\right]
$$

$Q_{r o}^{*} \quad$ é calor cedido pelo radiador ao ambiente.

$\alpha_{r}\left(v_{v}\right)$ é calculada segundo a equação (12);

$v_{v} \quad$ é a Velocidade do veículo;

$A_{r} \quad$ é a Área de contato entre o radiador e o ambiente;

$\vartheta_{r} \quad$ é a Temperatura média do radiador;

$\vartheta_{a} \quad$ é a Temperatura ambiente;

Considerando que o radiador está em equilíbrio térmico com o líquido refrigerador o atravessando, pode-se fazer:

$$
\vartheta_{r}=\frac{\vartheta_{r i}+\vartheta_{r o}}{2}
$$

Desprezando as trocas de calor nos dutos, temos, aonde a equação (26) é uma relação a ser identificada posteriormente:

$$
\begin{gathered}
\vartheta_{r i}=\vartheta_{e o} \\
\vartheta_{r o}=f\left(\vartheta_{e i}, \vartheta_{e o}, \dot{m}_{r}, m_{b y}\right)
\end{gathered}
$$


$\vartheta_{e o}$ é a notação simplificada de $\vartheta_{\text {eng out }}$.

$\vartheta_{\text {ei }} \quad$ é a notação simplificada de $\vartheta_{\text {eng,in }}$.

$m_{b y}$ é o fluxo do fluido de refrigeração que circunda paralelamente ao duto do radiador no circuito de refrigeração conforme Figura 5.

$\dot{m}_{r} \quad$ é o fluxo do fluido de refrigeração que passa através do radiador.

A temperatura $\vartheta_{e i}$ pode ser modelada, considerando os atrasos devidos, como a temperatura do ponto de confluência do líquido de saída do refrigerador com aquele que trafegou paralelamente ao mesmo. A temperatura $\vartheta_{\text {mix }}(t)$ do ponto de confluência é formulada conforme abaixo:

$$
\vartheta_{\text {mix }}(t)=\frac{1}{m_{r}^{\prime}(t)} \times\left[m_{r}(t) \times \vartheta_{r o}\left(t-\tau_{5 \rightarrow 4}\right)+m_{b y}(t) \times \vartheta_{\text {eo }}\left(t-\tau_{1 \rightarrow 4}\right)\right]
$$

Aonde $\tau_{X \rightarrow Y}$ é o atraso de transporte referente ao tempo que demora para o fluido de refrigeração ir do ponto $\mathrm{X}$ até o ponto Y. Para calcular este atraso, temos:

$$
\tau_{X \rightarrow Y}=\frac{l_{X \rightarrow Y}}{v_{X \rightarrow Y}}
$$

$l_{X \rightarrow Y}$ é a distância que o fluido de refrigeração percorre entre os pontos $\mathrm{X}$ e Y;

$v_{X \rightarrow Y}$ é a velocidade do fluido de refrigeração entre os pontos $\mathrm{X}$ e Y.

A velocidade do fluido de refrigeração entre os pontos X e Y pode ser calculado como:

$$
v_{X \rightarrow Y}=\frac{m_{X \rightarrow Y}^{*}}{\rho_{m} \cdot A_{X \rightarrow Y}}
$$

$\rho_{c} \quad$ é a densidade do fluido de refrigeração (use a densidade da água).

$m_{X \rightarrow Y}^{*}$ é o fluxo mássico de fluido de refrigeração entre os pontos $\mathrm{X}$ e Y.

$A_{X \rightarrow Y}$ é a seção transversal do duto entre os pontos $\mathrm{X}$ e Y.

Relacionando $\vartheta_{\text {mix }} \operatorname{com} \vartheta_{\text {ei }}$ :

$$
\vartheta_{e i}(t)=\vartheta_{m i x}\left(t+\tau_{4 \rightarrow 6}\right)
$$

\section{SISTEMA DE REFRIGERAÇÃO}

\subsection{Bomba do fluido de refrigeração (bomba d'água)}

Para o caso típico onde temos uma bomba mecânica, existe uma relação direta entre a velocidade do motor e o fluxo do fluido de refrigeração, conforme equação (31). Esta relação pode ser obtida junto ao fabricante ou em ensaios feitos em bancada como ilustra a figura $6[1]$.

$$
\dot{m}_{c}=K \cdot \omega_{e}
$$




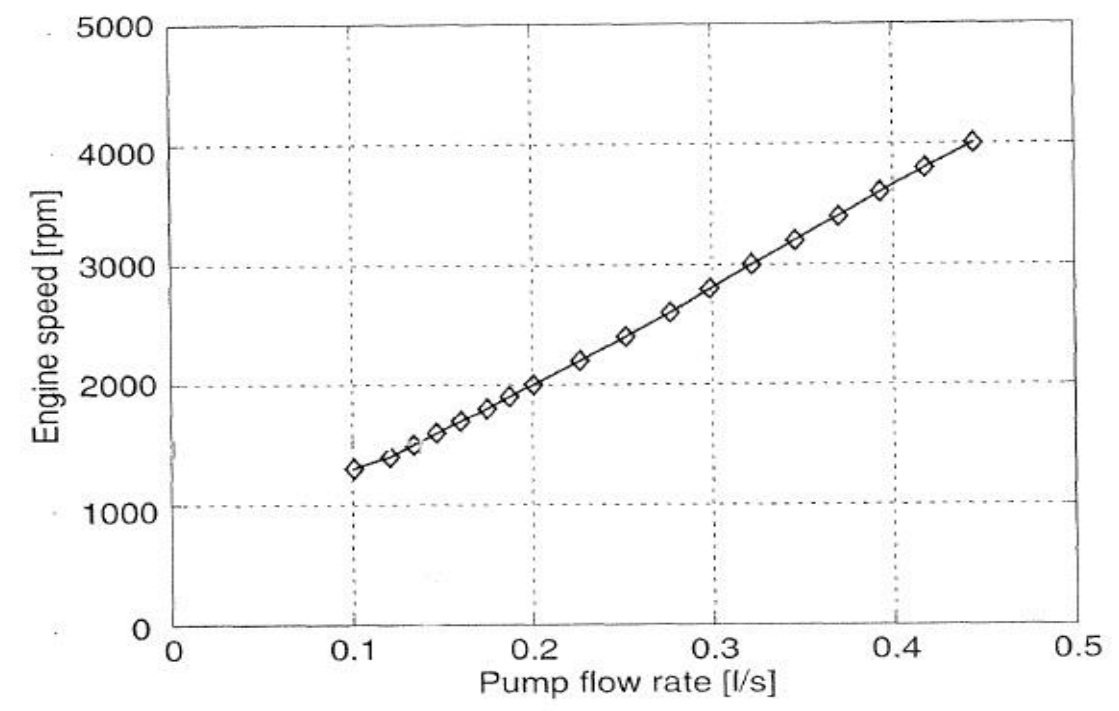

Figura 6: Relação entre a velocidade do motor e a vazão volumétrica do fluido de refrigeração para uma bomba mecânica típica. Fonte: [1]

\subsection{Válvula termostática}

A válvula termostática regula a porção do fluido refrigerante que vai para o radiador conforme a temperatura do fluxo, garantindo que o motor tenha refrigeração adequada. Possui acionamento termomecânico através de expansão térmica de seus elementos internos [1][11]. A relação entra a abertura da válvula termostática com a sua pode ser vista na figura 7 para uma válvula termostática específica.

A figura 8 apresenta a relação entre a abertura da válvula e vazão através do radiador. A partir da informação contida nas duas curvas é possível levantar um modelo da válvula termostática obtendo a relação entre temperatura do fluido e a vazão através do radiador, considerando que a válvula termostática está em equilíbrio térmico com o fluido refrigerador.

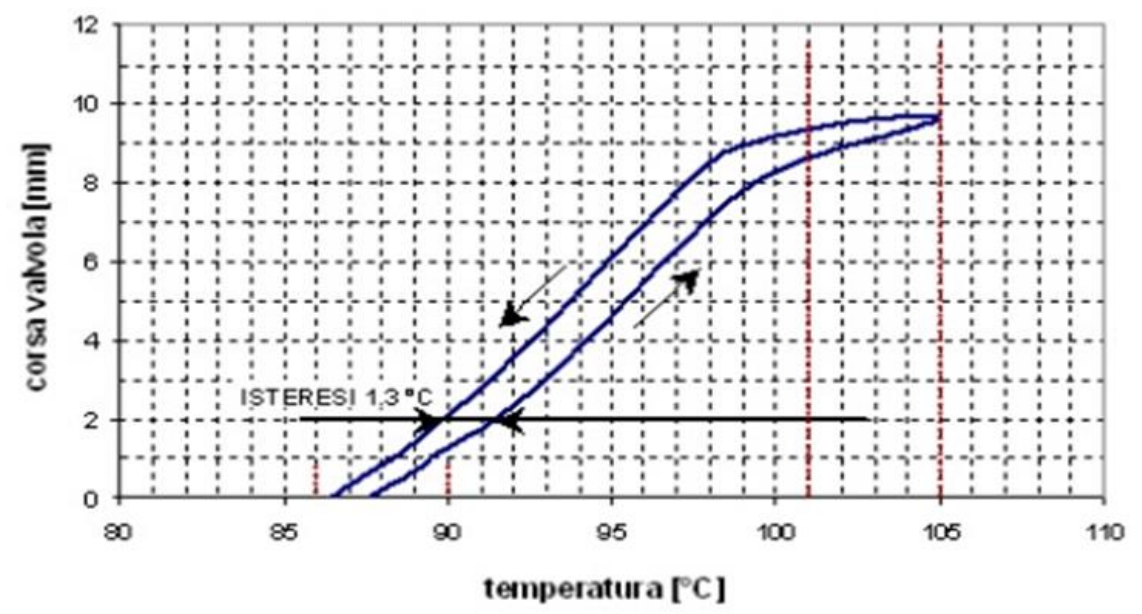

Figura 7: Abertura v.s. temperatura para uma válvula termostática genérica. Fonte: 


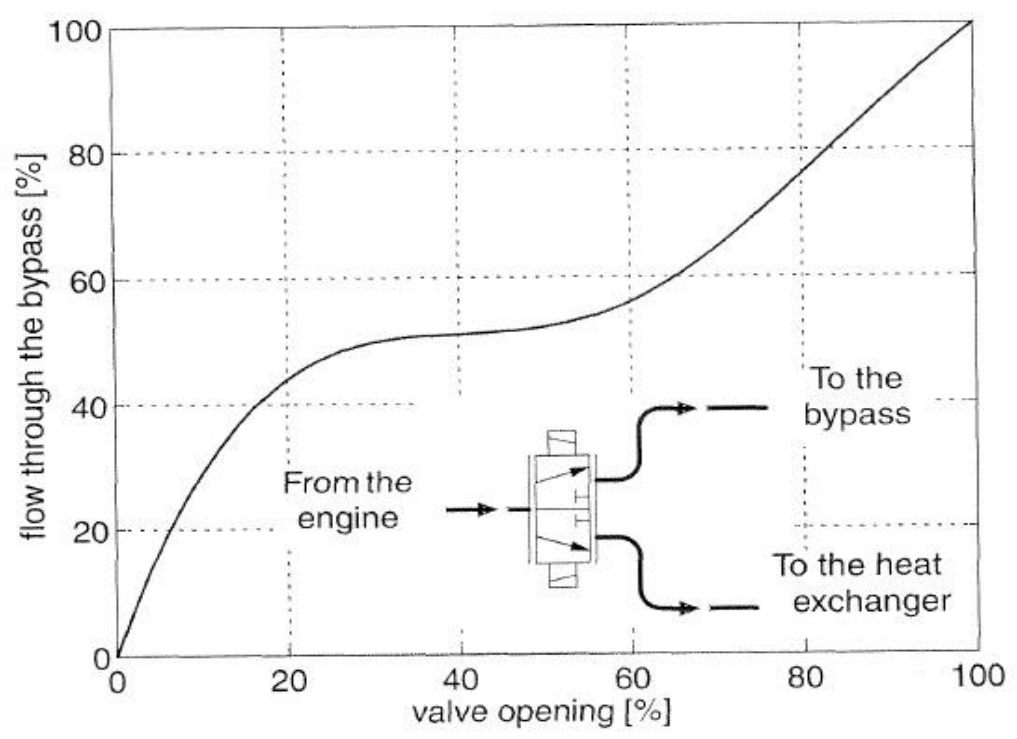

Figura 8: Fluxo v.s. abertura para uma válvula termostática genérica. Fonte: [1]

Pode-se aproximar o levantamento da figura 7 pela equação (32) considerando $82^{\circ} \mathrm{C}(355 \mathrm{~K})$ como valor genérico de temperatura na qual a válvula começa a abrir [12]. O valor de $\mathrm{K}$ pode ser obtido em ensaios utilizando um sensor de temperatura instalado na mangueira de saída do radiador, de forma a ser possível o uso do sistema (33), já que com tal sensor será conhecido $\vartheta_{r o}$.

$$
f\left(\vartheta_{\text {eo }}\right)=K \cdot\left(\vartheta_{\text {eo }}-355\right)
$$

$f\left(\vartheta_{\text {eo }}\right)$ é a abertura da válvula termostática.

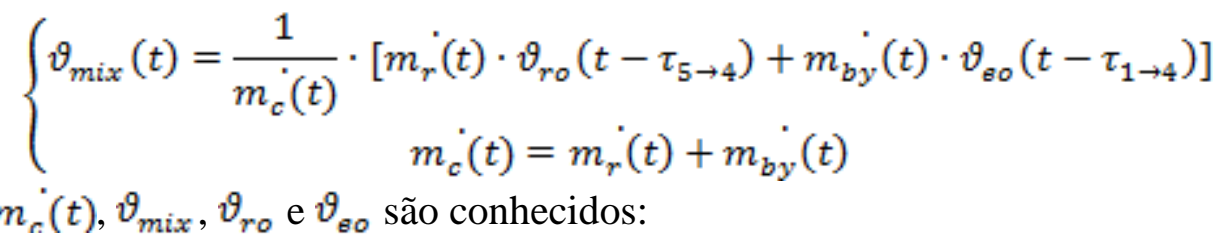

A partir do sistema (33), poderemos obter a razão $u_{1}$ presente no diagrama da Figura 10:

$$
u_{1}=\frac{m_{b y}^{\prime}(t)}{m_{n}^{\prime}(t)}
$$

\section{DIAGRAMAS ESTRUTURAIS DOS MODELOS TÉRMICOS}

Várias variáveis térmicas e mássicas expostas neste artigo podem ser encontrados com consultas a manuais do fabricante, tabelas físico-químicas, tabelas termodinâmicas, dentre outros. Entretanto, as variáveis as quais o pesquisador não conseguiu obter através destes meios, podem ser obtidas usando métodos de identificação que utilizam os diagramas expostos abaixo. Tais diagramas podem também ser usados como modelo final, a ser implementado em software de simulação ou em hardware de prototipagem. 


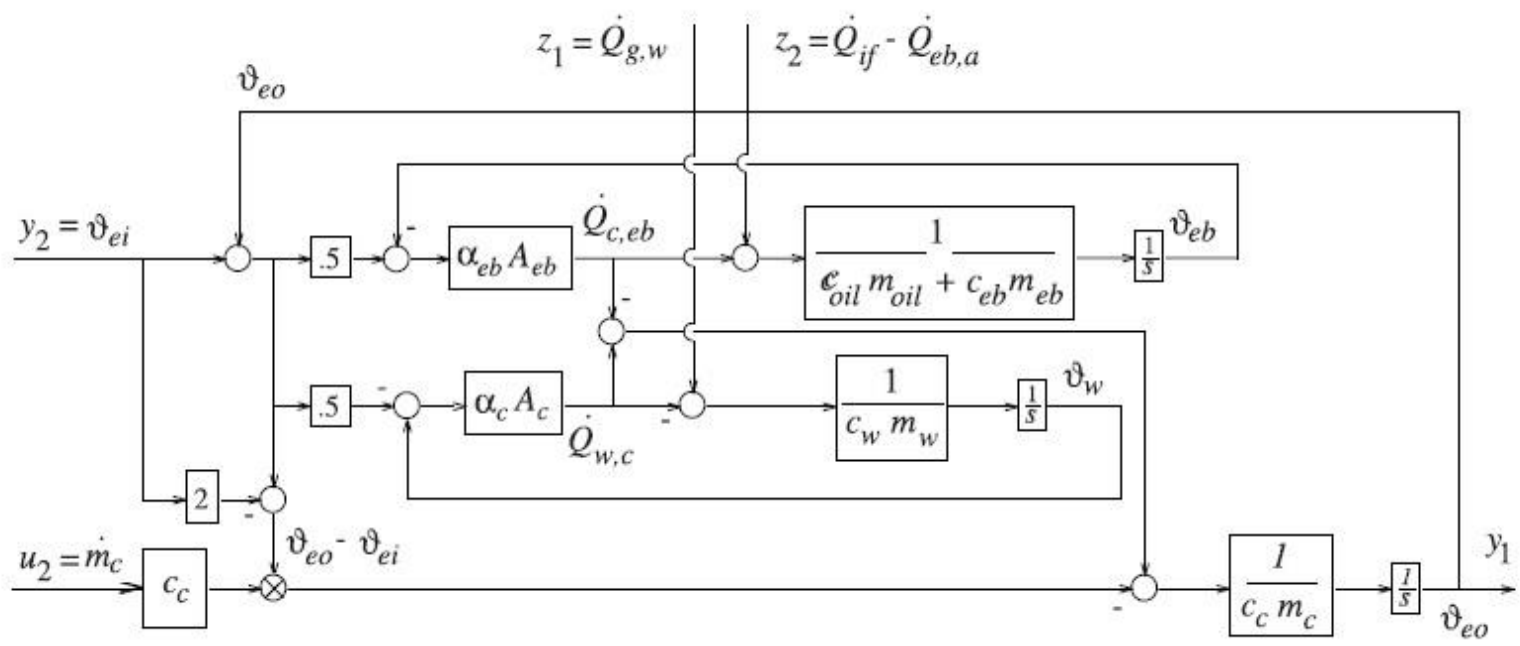

Figura 9: Diagrama estrutural do modelo térmico interno. Fonte: [4]

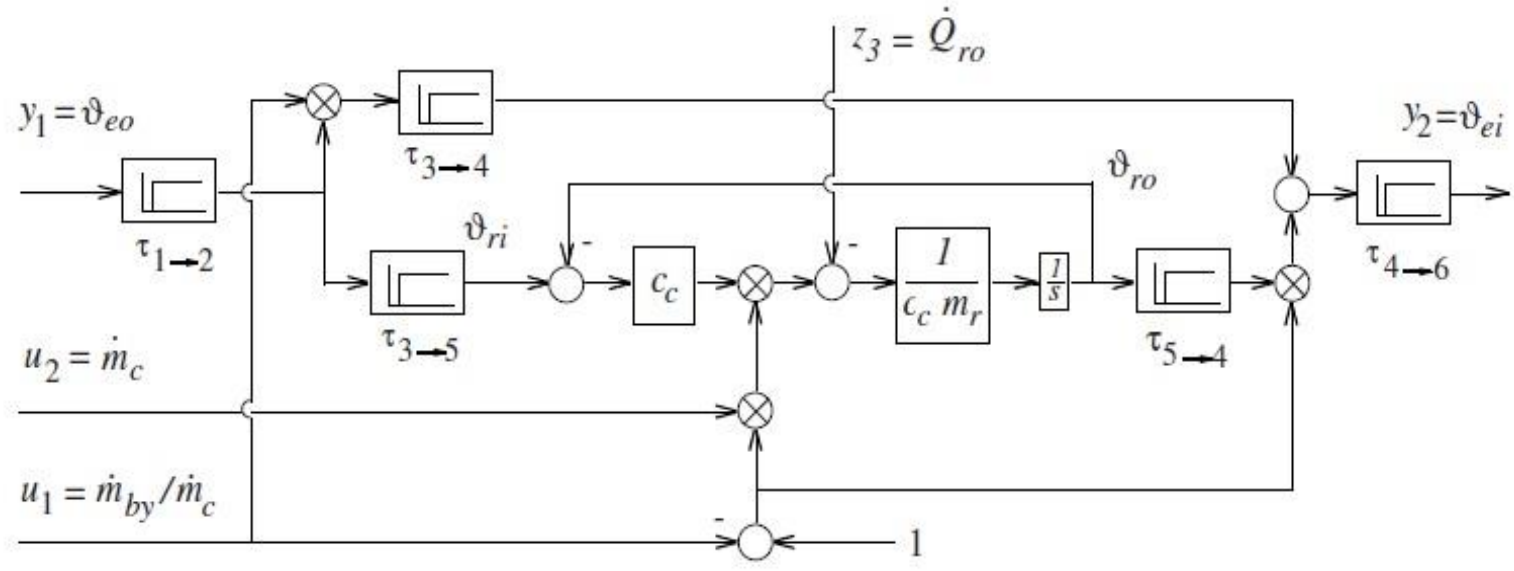

Figura 10: Diagrama estrutural do modelo térmico externo. Fonte: [4]

\section{CONCLUSÃO}

Neste artigo foi abordado a formulação matemática da modelagem dos diversos sistemas térmicos que compõem o motor de combustão interna do veículo, demonstrando como a energia proveniente da combustão se distribui pelo motor desde o momento de sua geração, produzindo energia mecânica e o restante sendo rejeitado ao meio ambiente. Procurou-se, conjuntamente, oferecer uma explicação física dos processos envolvidos.

Esta modelagem é útil para a elaboração de sistemas de controle que visem controlar a temperatura do motor, com fins diversos como eficiência.

As modificações e análises necessárias a serem feitas para o modelo de sistemas térmicos com o uso de tecnologia flex, numa comparação relativa ao uso de monocombustível, foram o acréscimo de uma variável a mais na estimativa da entalpia dos gases de entrada e de saída, que agora dependem da composição variada, assim como a interface sempre presente desta modelagem com os modelos dos sistemas mecânicos, nos quais protagonizam grandezas e medidas altamente correlacionadas com a composição, como a pressão média líquida 
equivalente e a eficiência, o que proporciona a influência da composição variada em todo o fluxograma de calor, conforme visto neste artigo.

\section{REFERÊNCIAS}

[1] KANNE, Elena Cortona; Engine thermomanagement for fuel consumption reduction. 2000. Tese de Doutorado. ETH Zurich.

[2] SKOGTJÄRN, På; Modelling of the exhaust gas temperature for diesel engines. Master Thesis. Linkopings Universitet. 2002.

[3] KANDYLAS, I. P; STAMATELOS, A. M. Engine exhaust system design based on heat transfer computation. Energy Conversion and Management, v. 40, n. 10, p. 1057-1072, 1999.

[4] GUZZELLA, Lino; ONDER, Christopher; Introduction to modeling and control of internal combustion engine systems. 2. ed. Springer Science \& Business Media, 2010.

[5] ALRAYYES, Taleb; The effect of ethanol-gasoline blends on SI engine energy balance and heat transfer characteristics. 2011. Tese de Doutorado. University of Nottingham.

[6] UNKNOWM; Universidade de Sevilla, departamento de física aplicada III, Ciclo Otto. Disponível em:

http://laplace.us.es/wiki/index.php/Ciclo_Otto. Acesso em: maio de 2017.

[7] ISERMANN, Rolf; Engine Modeling and Control; Springer. First Edition. 2014. 637p.

[8] SHUDO, Toshio; SUZUKI, Hiroyuki; Applicability of heat transfer equations to hydrogen combustion. Jsae Review, v. 23, n. 3, p. 303-308, 2002.

[9] HOHENBERG G; Experimentelle Erfassung der Wandwärme von Kolbenmotoren, Habilitation, Technische Universität Graz, 1980

[10] SANTOS, José Carlos Oliveira et al; Estimativa da capacidade calorífica de óleos lubrificantes automotivos por DSC. Terceiro Congresso Brasileiro de P\&D em Petróleo e Gás. 2004.

[11] SCUOLA di Dottorato di Ricerca; Engine Vehicle Integration - Exhaust system. 2010. Disponível em: http://slideplayer.com/slide/614650/. Acesso em: Maio de 2017.

[12] VDOVIN, Alexey; Cooling performance simulations in GT-Suite. Master's Thesis. Department of Applied Mechanics. Chalmers University of Tecnology. Sweden. 2010. 